\title{
¿Comunicación responsable? El caso de una organización educativa
}

\section{Responsible communication? The case of an educational organization}

\author{
Alfredo Tapia-Carreto* \\ Hilda Gabriela Hernández Flores \\ Paola Eunice Rivera Salas \\ doi.org/10.29043/liminar.v20i2.913
}

Resumen: Con el objetivo reconocer las discrepancias entre los preceptos establecidos por la perspectiva en comunicación responsable y la práctica comunicativa de una organización educativa, se utilizó un método de Escalas Semánticas Diferenciadas sobre una muestra definida por criterios señalados por el Instituto de Socioeconomía de las Empresas y de las Organizaciones (ISEOR) respecto a su modelo de intervención para encontrar costos ocultos. Se concluye en un análisis acerca de la importancia de una buena práctica comunicacional en las organizaciones, tras hallar discrepancias entre lo señalado en la perspectiva y la práctica de la comunicación responsable en la organización objeto de estudio.

Palabras clave: comunicación responsable, comunicación organizacional, comunicación institucional, intervención organizacional, instituciones educativas.

Abstract: Responsible Communication perspective and the communicative practice of an educational organization, we used a method of Differentiated Semantic Scales on a sample, using criteria defined by the Institute of Socio-Economy of Companies and Organizations (ISEOR) in its intervention model to find hidden costs. We conclude by analyzing the importance of having good communication practices in organizations, after finding discrepancies between what is stated in the perspective and the actual practice of responsible communication in the organization under study herein.

Key words: responsible communication, organizational communication, institutional communication, organizational intervention, educational organizations.

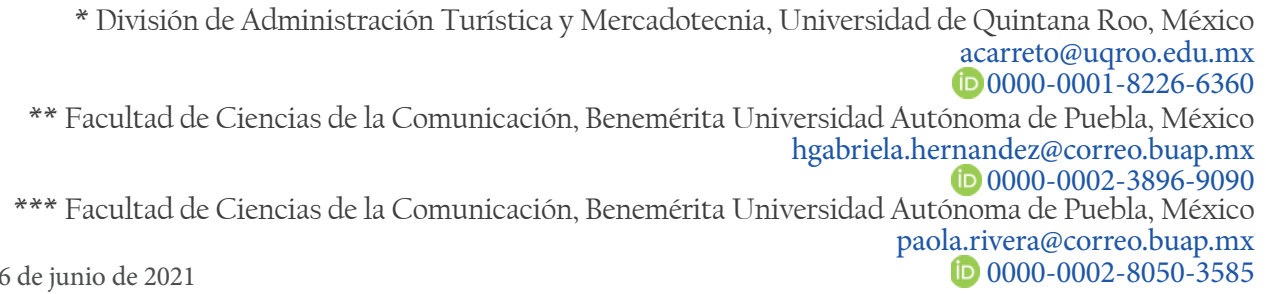

Recibido: 16 de junio de 2021

(iD) 0000-0002-8050-3585 


\section{Introducción}

$\mathrm{E}$ 1 propósito del proyecto que dio origen a este artículo fue reconocer las discrepancias entre los preceptos establecidos por la perspectiva emergente de la comunicación responsable y la práctica comunicativa de una organización educativa, para lo cual se ahondó en la divergencia entre un ideal teórico y una práctica cotidiana de gestión comunicativa. La importancia de estrechar dicha divergencia recae en el impacto poco explorado de la comunicación en relación con los valores intangibles de una organización, como lo son su imagen y su reputación corporativa (Orozco-Toro y Ferré-Pavia, 2019).

La investigación ocupó como principal referencia teórica la perspectiva de la comunicación responsable, grosso modo entendida como:

[...] el diálogo comprometido entre la organización y sus públicos, que se espera honesto, congruente a las acciones y, por ende, responsable, por lo que establece una gestión comprometida, no sólo desde la marca, el producto o el servicio que se presta, sino con todo lo que se comunica. Cuando la organización alcanza este tipo de comunicación, entonces y sólo así, logra una relación de confianza, que asegura la sintonía y participación de los públicos generando una reputación sostenida, aún en situaciones complejas de comunicación pública (Hernández Flores, 2018).

Cabe señalar que la comunicación responsable surge estrechamente relacionada con diferentes subcampos de la comunicación, uno de ellos el organizacional, siendo este tipo de comunicación una herramienta estratégica que pone de manifiesto, tanto dentro como fuera de las organizaciones, la creación de vínculos estables y de legitimidad social (Paladines, Valarezo y Yaguache, 2013) con sus públicos, que en la organización escolar son: estudiantes, docentes, personal administrativo y operativo y, dependiendo del grado escolar, jefes y jefas de familia, así como sociedad. Este tipo de comunicación se deriva, a su vez, de la teoría clásica de la comunicación (ver Figura 1), cuyos preceptos funcionales son: emisor, mensaje, canal, receptor, interferencia y retroalimentación.

Figura 1. Modelo clásico de comunicación

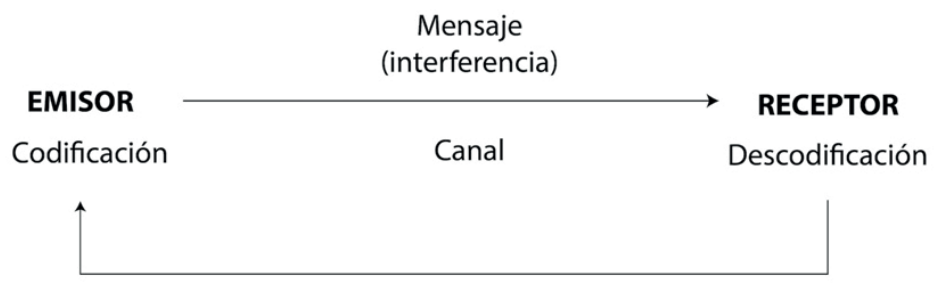

Fuente: elaboración propia.

La comunicación responsable, adicionalmente, se sustenta en principios éticos que sin duda remiten al concepto aparentemente básico de "responsabilidad" (Capriotti, 1999; Fundación SERES, 2014; 
Rincón et al., 2019); sin embargo, solo hasta fechas recientes comenzó a complementarse el concepto de comunicación con el término de responsable, y la complejidad que implica la unión de ambas nociones puede constatarse cuando la responsabilidad se transversaliza en las subdisciplinas de la comunicación como un elemento indispensable de hacer comunicación.

\section{Marco teórico}

En una revisión de la literatura se observó que en pocos trabajos se retoma la comunicación responsable como foco principal. Sin embargo, es pertinente señalar que, en las últimas décadas, la preocupación por atender la responsabilidad en los recursos de comunicación ha sido constante, como se observa en el caso de Jaksa (1996), quien analiza los riesgos de comunicación que se ven involucrados en el uso de nuevas tecnologías en el panorama globalizado. Por su parte, Villalba (2015) explica que la comunicación responsable tiende a confundirse con las acciones informativas que caracterizan la "responsabilidad social"; sin embargo, la diferencia entre ambos conceptos comenzó a marcarse cuando autores como Remund (2015) describieron la comunicación responsable haciendo énfasis en la gestión de la comunicación basada en los valores éticos demostrados en las acciones comunicativas. Así, la importancia de este tipo específico de comunicación en las organizaciones recae en su capacidad de gestión desde una mirada ética, y no únicamente desde su capacidad comunicacional para corresponder a los públicos y grupos de interés de la mano de la responsabilidad social organizacional (Rincón y Ramírez, 2018).

Al respecto, Pertúz (2018) mencionó la importancia de analizar las bases culturales y éticas de los problemas que se presentan en el día a día de las organizaciones, así como la necesidad de abordar estas situaciones particulares mediante paradigmas emergentes, entre otros, aquellos relacionados con la comunicación.

En este contexto, la comunicación responsable ofrece a las organizaciones la posibilidad de un manejo más consciente de sus sistemas comunicativos y de sus entornos de influencia, lo que les permite establecer un diálogo con los públicos basado en los rasgos de la perspectiva: honestidad, transparencia, empatía, interactividad y consistencia, situación que se extiende hasta sus políticas internas, su cultura y su comportamiento organizacional. Con ello, se estructura a mediano y largo plazo una relación de confianza y compromiso que asegura una comunicación abierta, fluida y de retorno, lo que contribuye a que la organización estudiada sea más aceptada en su entorno porque, acorde a ello, comunica responsablemente.

Sin embargo, la comunicación responsable, como concepto holístico y transversal, admite la capacidad de adaptarse a los distintos subcampos de la comunicación en los que se enmarca una organización, una profesión o un medio comunicativo. Así, los cinco rasgos mencionados anteriormente se consideran los rasgos básicos de la perspectiva, a los cuales se suman para su mejor comprensión los rasgos específicos que corresponden al tipo de subcampo, en este caso el corporativo e institucional. En nuestra investigación en específico, hicimos especial énfasis en la comunicación institucional, entendida esta como la comunicación que emana de la organización hacia los públicos a través de cualquier forma o herramienta comunicativa, congruente con la filosofía institucional y que, por ende, deja entrever una gestión responsable de la marca, el discurso, el producto o el servicio, logrando así legitimidad y reputación comunicativa (Rivera-Salas y Hernández-Flores, 2020). A este subcampo en particular se agregan los rasgos particulares del mismo: imagen, reputación y cultura organizacional (ver Figura 2). 
Figura 2. Subcampo y rasgos de la comunicación responsable

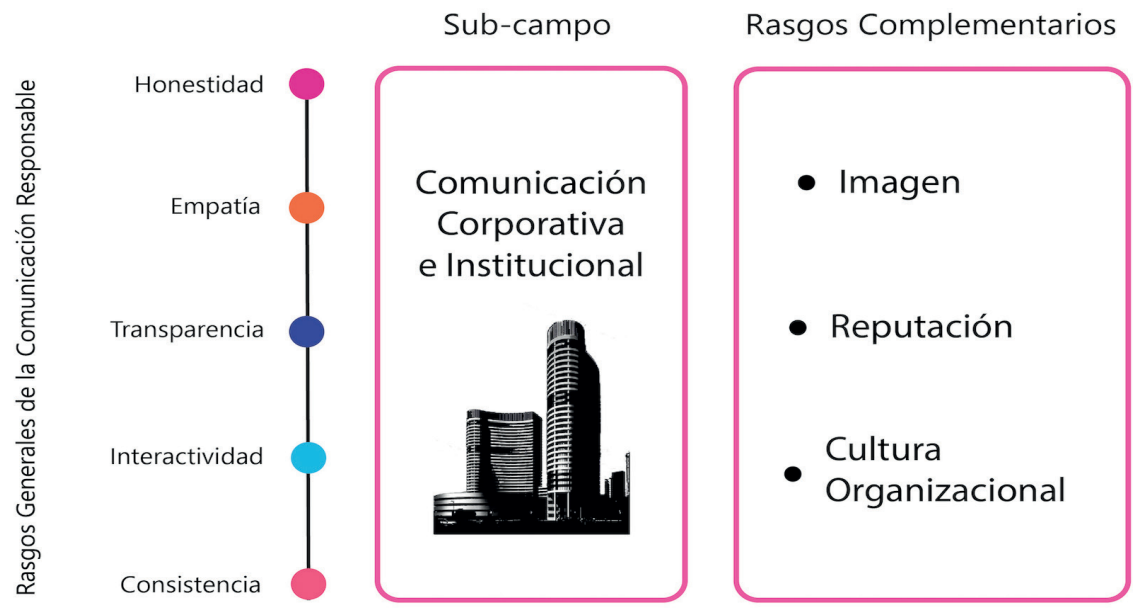

Fuente: elaboración propia.

En la organización, la comunicación debe poseer un enfoque holístico y cumplir con los rasgos básicos referidos de la comunicación responsable: honestidad, empatía, transparencia, interactividad y consistencia, los cuales enriquecen o sustentan el diálogo con los grupos de interés, bajo una retroalimentación continua (interactividad), que en este caso permite alcanzar las metas de la organización. Así, el proceso tradicional se complementa a través de los aspectos mencionados (ver Figura 3).

Figura 3. Modelo de comunicación responsable

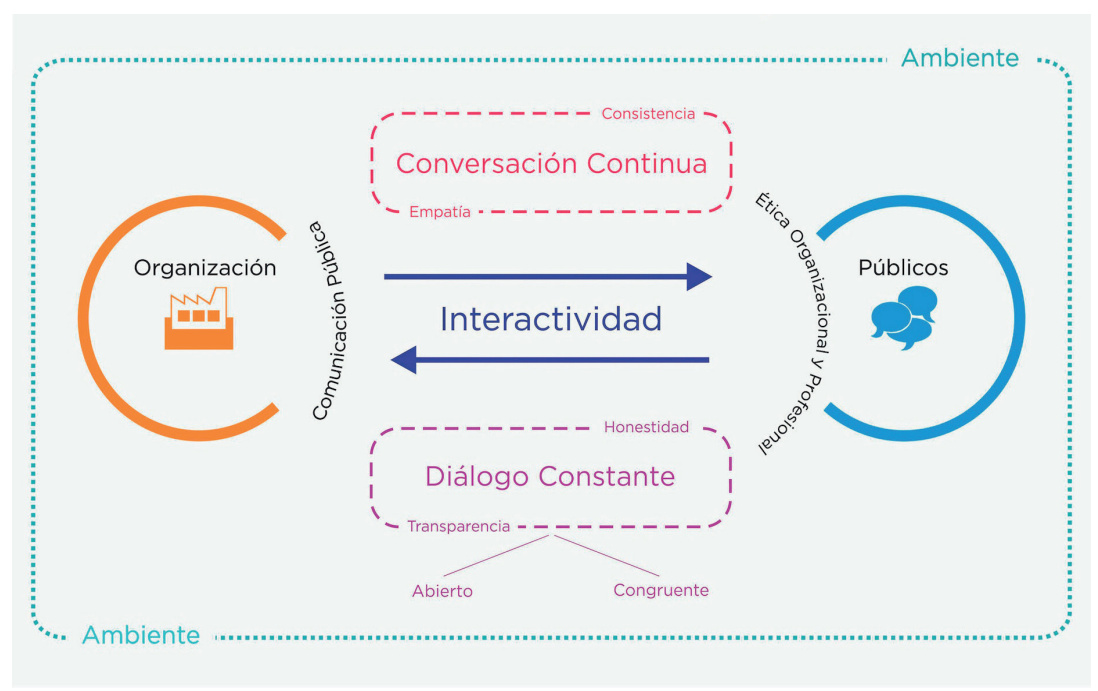

Fuente: Hernández Flores (2020). 
En la Figura 3 se representa el esquema modelo de comunicación responsable, donde interactúan los elementos básicos de este tipo de comunicación, que a continuación se definen:

1. Honestidad: se refiere a la posibilidad de verificar lo que comunica una organización a través de datos e información oficial, así como las referencias proporcionadas por el emisor.

2. Empatía: implica mostrar respeto por la audiencia al considerar su contexto y necesidades específicas en el momento en que se expresa la comunicación institucional.

3. Transparencia: significa aportar toda la información necesaria sobre lo que hace la organización y cómo lo hace.

4. Interactividad: involucra una comunicación dialógica en la que no solo se considera lo que la organización tiene que decir, sino también la retroalimentación que recibe de sus audiencias y su reconstrucción a partir de ella.

5. Consistencia: independientemente de las diferentes audiencias con las que una organización se comunica, es importante mantener un discurso institucional coherente que vincule lo que se comunica con la cultura organizacional, especialmente con sus valores organizacionales.

De acuerdo con la Fundación Sociedad y Empresa Responsable (Fundación SERES, 2014), las funciones de la comunicación en el contexto de los cinco rasgos anteriores son diez:

1. Coordinación de la comunicación: en muchas ocasiones los departamentos o áreas o de la organización, o incluso su tamaño, no facilitan que se lleve a cabo una coordinación óptima. El área de comunicación es la principal aliada para la difusión de las estrategias y acciones dentro de la organización, además de que es importante incorporar un Plan de Comunicación Responsable (PCR) que comunique las acciones en congruencia con el mismo.

2. Alinear objetivos: para definir un PCR resulta necesario repasar los objetivos, metas y estrategias de la organización desde su planteamiento ético. Como reflexión previa, antes de comunicar ayuda contestar las siguientes preguntas: ¿qué hacemos?, ¿por qué lo hacemos?, ¿para qué lo hacemos?, ¿cómo ello incide en nuestros públicos internos y externos? Estas interrogantes deben responderse siempre antes de emitir mensajes o información, lo que contribuirá a generar una comunicación más responsable y asertiva.

3. Defender un modelo participativo: establecer el modelo de comunicación responsable en el trabajo interno también es positivo. Se deben contemplar todas las vías de participación de los equipos o colaboradores de la organización. Para ello, se valora como necesario capacitar al personal sobre el modelo y sus rasgos, y a partir de ello organizar encuestas, grupos de trabajo y comités de participación para que el personal esté involucrado en los compromisos de la organización en los diferentes ámbitos —-social, económico y ambiental—y se identifique con los proyectos. Otra opción es "promover la comunicación responsable a través del voluntariado", porque un modo adecuado de de que el personal integre los mecanismos de comunicación y los practique consiste en que se implique de manera simbólica a través del voluntariado.

4. Activar todos los canales: ¿con qué canales cuenta la organización para poner en práctica una comunicación responsable? De nuevo se hace hincapié en la necesidad de establecer 
una alianza y conexión con el Departamento de Comunicación para asegurar que todos los canales dentro de la organización están activados y dispuestos a comunicar responsablemente con el propósito de llegar a todo el equipo y globalizar la perspectiva comunicativa desde la responsabilidad en todos los medios: Internet, revista corporativa, email, cartelería, pantallas digitales, paneles, etcétera.

5. Segmentar: para definir los contenidos de información que se desean comunicar es importante definir el público objetivo al que va dirigido el mensaje. De este modo, se conseguirá mayor impacto al combinar información, emoción y canal de distribución. El mensaje además debe estar alineado con la marca, entendiendo que diferentes marcas permiten diferentes tipos de comunicación.

6. Apoyarse en claves de la comunicación responsable: como en cualquier acción de este tipo de comunicación es importante introducirse en la posición de las personas destinatarias y tratar de manejar claves de marketing para maximizar el impacto: acciones teaser (para generar intriga en el público) y soportes innovadores (dispositivos gadgets) basados en la ética y la responsabilidad.

7. Incorporar lenguaje audiovisual: hoy en día la comunicación es eminentemente audiovisual, un lenguaje que resulta más atractivo y ágil y que fomenta el recuerdo en la mente de los individuos. Esto incrementa el impacto del mensaje dentro y fuera de la institución.

8. Comunicar a través del ejemplo: es imprescindible y muy efectivo asegurarse de dar voz y espacio a los equipos que más activamente colaboran con la política de comunicación responsable de la organización en todas las acciones y canales.

9. Favorecer la retroalimentación: las aportaciones más valiosas proceden de quienes viven el día a día del trabajo de la organización, por lo que es importante contar con canales de comunicación responsable que aseguren el feedback de los equipos sobre la estrategia y las acciones comunicativas internas.

10. Medir resultados: lo que no se mide no existe, por lo que es básico medir las acciones de comunicación responsable atendiendo los siguientes criterios:

- Seguimiento: ¿cuánta gente ha leído las informaciones emitidas?, ¿cuántas sugerencias o dudas se reciben?

- Participación: ¿cuánta gente ha participado en foros, grupos, comités?, ¿cuánta participa en las acciones y programas de voluntariado?

- Opinión y valoración: gestionar con las áreas correspondientes (comunicación, recursos humanos) la inclusión de encuestas de comunicación y de clima laboral para conocer la valoración que los equipos otorgan a la política y las acciones de comunicación responsable de la organización (Fundación SERES, 2014).

En tal sentido, la comunicación responsable debe atender las necesidades y expectativas de los grupos de interés, así como compartir valores, creencias, acciones, políticas y estrategias, para crear vínculos que ayuden a consolidar la relación con sus stakeholders (Meseguer, García y Hernández, 2018).

Por su parte, la organización debe involucrar a sus colaboradores y colaboradoras en esta modalidad de comunicación porque esto contribuirá a que desarrollen liderazgo, ética, responsabilidad y solidaridad, y a que se involucren en lo que sucede en su entorno. En este sentido, la comunicación se debe gestionar desde los valores institucionales, y cada persona colaboradora, además de los grupos de interés, de la organización deben ser partícipes en darla a conocer. 
En cuanto a la "organización escolar", en México esta se ha caracterizado por un "limitado enfoque de planificación de una reforma, un cambio general o una innovación particular, soliendo poner mucho énfasis en cuestiones metodológicas, de formación docente, de diseño curricular, de evaluación de los aprendizajes" (Tapia-Carreto, Mendoza y Martínez, 2016). Esto ocurre principalmente en las escuelas de niveles básicos, tal vez debido a que en México los elementos mencionados mantienen un estatus de obligatoriedad. Cabe resaltar que, tal y como afirma Fuentes, "La Organización Escolar adquiere una mayor utilidad y sentido cuando nos movemos en realidades complejas, donde la necesidad de ordenación es importante dada una supuesta mayor dispersión de intereses individuales" (2015, p. 1).

La universidad, en tanto una organización escolar, también se estudia bajo esos tratamientos. Complementariamente, consideramos las organizaciones escolares como una red de múltiples encuentros, y reconocemos también que:

[...] se enfrentan, en forma constante, a distintos y complejos desafíos por las demandas que el dinámico y desafiante contexto social exige de ellas como organizaciones productoras del conocimiento mediante distintos procesos como la formación de profesionales y la investigación, como parte de su responsabilidad social a la cual se deben (Garbanzo-Vargas, 2016, p. 76).

En este marco, nuestro objeto de estudio es una universidad pública, específicamente la Unidad Académica Cozumel de la Universidad de Quintana Roo, donde se observó el ejercicio de la comunicación institucional formal de cualquier forma en que esta fue generada, en el lapso memorístico de los testimonios obtenidos de las personas entrevistadas.

Por su parte, el modelo de "gestión socioeconómica" creado por el Dr. Savall en 1974 y que desde entonces ha desarrollado junto con su equipo de investigación del Instituto de Socioeconomía de las Empresas y Organizaciones (ISEOR), mediante una interactividad cognitiva con más de mil organizaciones ubicadas en 31 países diferentes, muestra una teoría y una metodología que asocian la consultoría con la investigación y la formación para construir alternativas frente a la problemática de la gran diversidad de organizaciones: desde las microempresas productoras de bienes y servicios, hasta oficinas gubernamentales, incluyendo instituciones educativas. Se decantan principios genéricos a partir de lo contingente de cada caso (ISEOR, s. f.).

Derivado de lo anterior, en el proyecto origen de este artículo nos planteamos cuestionamientos como los siguientes: ¿cómo se produce la comunicación en una organización educativa?, ¿cómo ocurre este proceso en la organización escolar en la que laboramos?, ¿ esta comunicación ocurre de forma responsable o, por el contrario, ignora los preceptos básicos de la responsabilidad?, ¿cómo podemos reconocer las discrepancias entre los preceptos establecidos por la perspectiva de comunicación responsable y la práctica comunicativa de nuestra organización?

\section{Marco metodológico}

Para alcanzar el objetivo planteado se aprovechó una intervención organizacional en marcha en la Unidad Académica Cozumel de la Universidad de Quintana Roo para descubrir los costos ocultos de la organización educativa objeto de estudio. En esta intervención se ocupó la metodología propuesta por el ISEOR (Fernández y Savall, 2004), que consistió en una entrevista de presentación con la coordinación 
de la organización escolar objeto de estudio de una hora y media de duración y un diagnóstico en cuatro etapas: 1) Etapa cualitativa, consistente en: realización de tres entrevistas con personal directivo para identificar disfuncionamientos; realización de nueve entrevistas de grupo con personal de base para identificar los disfuncionamientos; selección de alrededor de 239 frases testimonio; clasificación de frases testimonio en temas, categorías e ideas principales y cálculo de frecuencias; y elaboración de una matriz de convergencias-especificidades. 2) Etapa de los costos ocultos, consistente en: selección de los disfuncionamientos elementales dentro de las entrevistas realizadas en la parte cualitativa; realización de dos entrevistas de costos ocultos, e identificación de actos de regulación y cálculo de costos ocultos. 3) Etapa de elaboración, junto con el personal de jefatura, para llevar a cabo las matrices de competencia del personal a su cargo. 4) Reunión para el ejercicio del "efecto espejo".

Los elementos metodológicos utilizados en estas etapas fueron: entrevista de presentación, etapa cualitativa contemplada en la primera parte, selección de disfuncionamientos (particularmente los relativos a los procesos de comunicación), y realización de dos entrevistas más para delimitar costos ocultos, también con un enfoque de análisis comunicacional. A este enfoque se sumó el uso de las "escalas semánticas diferenciadas" de Zoltán Dörnyei (2007), que son similares a la escala Likert, en la que se utilizan varios ítems para evaluar un único objetivo, pero prestando especial atención al nivel de significación de las expresiones utilizadas.

Para la realización de las entrevistas se organizó el aparato gestor en dos grupos específicos de acuerdo con un orden jerárquico. De esta manera, los testimonios deberían recopilarse en forma similar, lo que permitiría organizar la colección de testimonios en perspectivas conjuntas, compartidas y coherentes con el grupo de trabajo entrevistado (ver Figura 4), de forma tal que se aseguraría la obtención de discrepancias entre las personas entrevistadas.

Figura 4. Arquitectura de la intervención

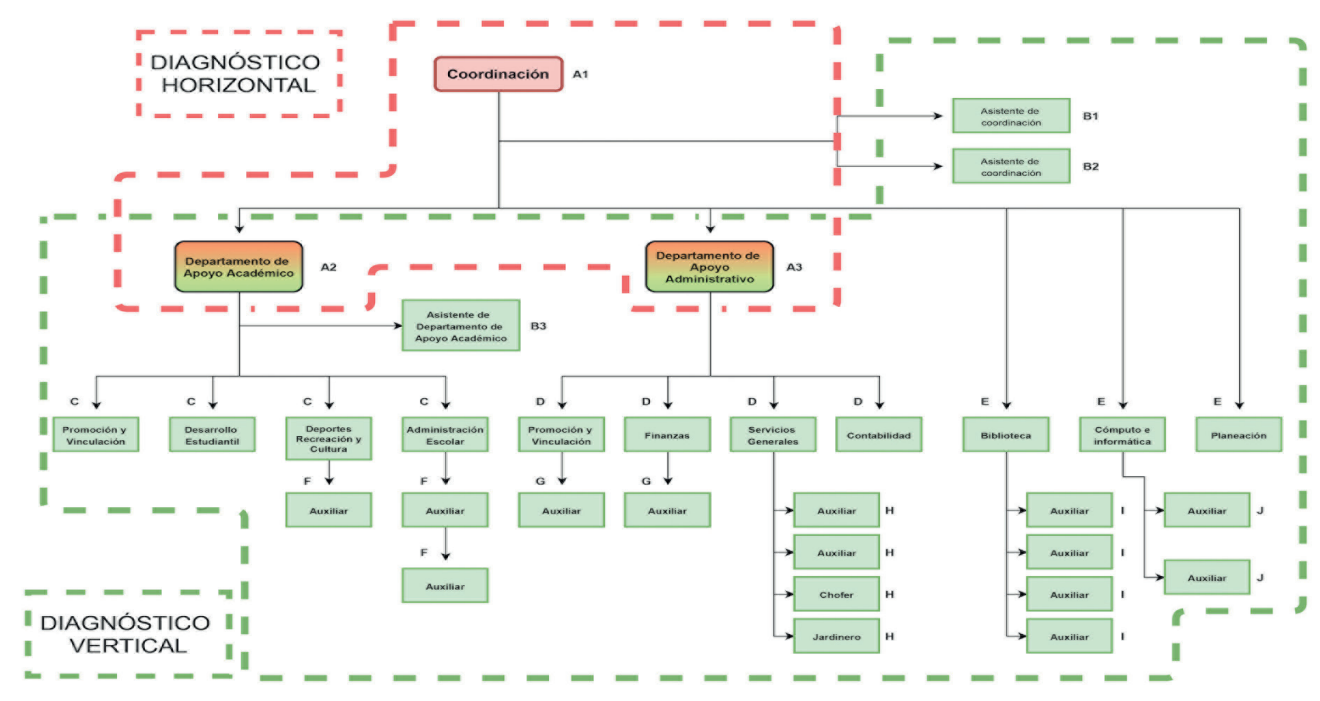

Fuente: elaboración propia. 
En este sentido, conviene recordar que, en un país como México, la fuerza laboral suele estar subyugada por las autoridades, y las autoridades suelen ejercer dominio autoritario sobre la fuerza laboral a su cargo, lo que deriva, entre otros factores, en perspectivas opositoras sobre el quehacer organizacional.

En este marco, las entrevistas se llevaron a cabo en dos subconjuntos, en una muestra de 31 gestores seleccionados, a manera de censo, de acuerdo con el tamaño de la institución, organizados en una jerarquía horizontal para estudiar el sector "jefaturas" y en una jerarquía vertical para estudiar el sector "personal base". Los grupos de formación-concertación se dividieron en tres sectores (ver Figura 5), y se llevaron a cabo un total de 15 entrevistas a 31 miembros de la organización: tres jefes, tres responsables de área (Biblioteca, Cómputo e Informática y Planeación), cuatro responsables de área del Departamento de Apoyo Administrativo (Recursos Humanos, Finanzas, Contabilidad y Servicios generales), cuatro responsables del Departamento de Apoyo Académico (Promoción y Vinculación, Desarrollo Estudiantil, Deportes, Recreación y Cultura, y Administración Escolar), dos asistentes de Coordinación, un asistente del Departamento de Apoyo Académico, dos auxiliares de Cómputo e Informática, tres asistentes de Biblioteca, un auxiliar de Recursos Humanos, un auxiliar de Finanzas, dos auxiliares de Servicios Generales, un chofer, un jardinero, dos auxiliares de Administración Escolar y un auxiliar de Deportes, Recreación y Cultura.

Figura 5. Grupos para la formación-concertación

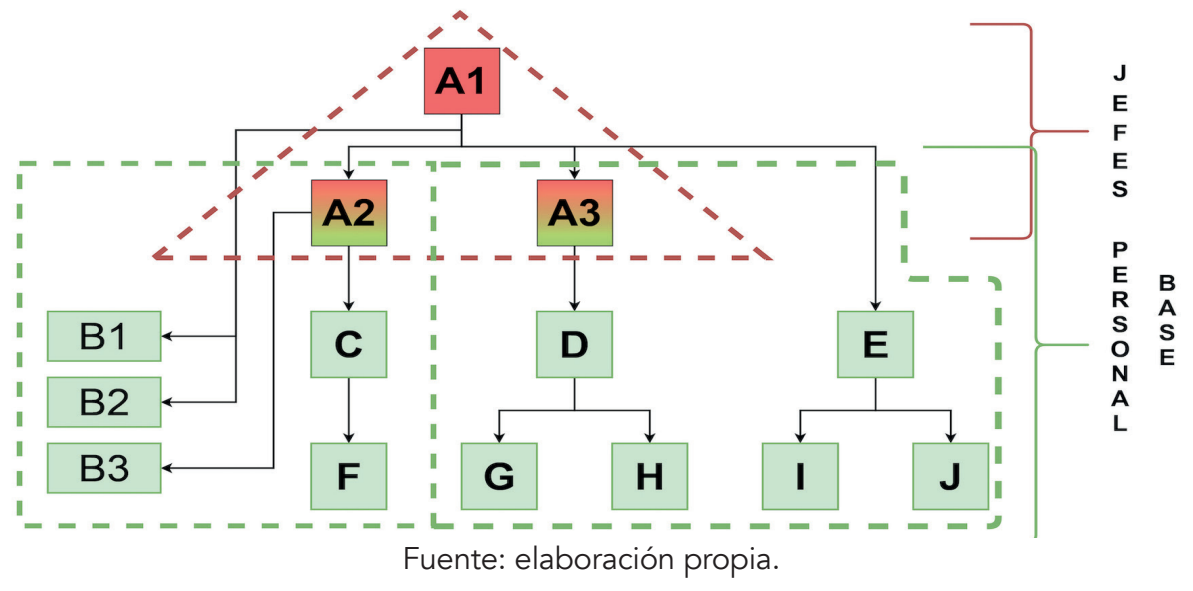

Los tres sectores en los que se entrevistó personal, entonces, fueron: el sector de autoridad (una coordinación y dos jefaturas), y dos sectores operativos (dos dependientes de las dos jefaturas).

\section{Técnicas e instrumentos de recogida de información}

En las entrevistas a profundidad, grupales e individuales, los instrumentos de recogida de información fueron una guía estructurada de cuestionamientos y la grabación de audio previa autorización de las personas entrevistadas. Los temas tratados en estas entrevistas fueron los sugeridos por el ISEOR en su metodología, siendo los principales: condiciones de trabajo, organización del trabajo, gestión del tiempo, comunicación-coordinación-concertación, formación integrada y aplicación estratégica. 
El diagnóstico se llevó a cabo en cuatro etapas. En la primera, una etapa cualitativa, se realizaron las quince entrevistas, se obtuvieron y clasificaron las frases testimonio en temas, categorías e ideas principales. La segunda parte correspondió a la etapa de los costos ocultos y en ella se realizó una selección de los disfuncionamientos elementales en las entrevistas efectuadas en la parte cualitativa, más dos entrevistas de costos ocultos, y se identificaron actos de regulación de los disfuncionamientos. En la tercera parte se elaboraron, junto con los jefes de departamentos, las matrices de competencias del personal a su cargo.

Finalmente se realizó una última reunión para efectuar el ejercicio del "efecto espejo". La parte de diagnóstico, que no se contempla en este documento por cuestiones de espacio, incluye la composición de la subpoblación de jefes de departamento, la composición de la subpoblación del personal de base del aparato administrativo, la función de las personas entrevistadas, el diagnóstico cualitativo (aglomeración de frases testimonio y matriz de convergencias y especificidades), un ejemplo de evaluación de costos ocultos y la matriz de competencias.

\section{Resultados y análisis}

Del total de entrevistas (tres individuales de hora y media con personal de jefaturas, más doce de grupo de entre hora y media y dos horas veinte minutos de duración, con el personal de base de mando medio y operativo), se seleccionaron de diecisiete a veintidós frases testimonio por entrevista - alrededor de trescientas en las quince entrevistas-. Estas frases testimonio se clasificaron en temas, subtemas e ideas clave, se ubicaron los extremos semánticos y se calcularon sus frecuencias. Se contrastaron contra nuestro ideal teórico - las diez funciones de la comunicación en el contexto de los cinco rasgos de la comunicación responsable-, y se realizaron las matrices de convergencias-especificidades; estas últimas, propias de un estudio secundario a publicarse en un momento posterior al que nos ocupa aquí. Con lo anterior, se diseñó una tabla que indica cuál de esas diez funciones se llevan a cabo, o no, en los tres sectores entrevistados, análisis que arroja el resultado que se muestra a continuación.

Tabla 1. Contraste entre ideal teórico y la práctica organizacional

\begin{tabular}{|c|c|c|c|}
\hline & Jefaturas & $\begin{array}{c}\text { Personal } \\
\text { de mando medio }\end{array}$ & $\begin{array}{l}\text { Personal } \\
\text { operativo }\end{array}$ \\
\hline Coordinación de la comunicación & $\mathbf{x}$ & $\mathbf{x}$ & $\mathbf{x}$ \\
\hline Alinear objetivos & $\mathbf{x}$ & $\mathbf{x}$ & $\mathbf{x}$ \\
\hline Defender un modelo participativo & $\mathbf{x}$ & $\mathbf{x}$ & $\sqrt{ }$ \\
\hline Activar todos los canales & $\mathbf{x}$ & $\sqrt{ }$ & $\mathbf{x}$ \\
\hline Segmentar & $\mathbf{x}$ & $\mathbf{x}$ & $\mathbf{x}$ \\
\hline Apoyarse en claves de la comunicación responsable & $\checkmark$ & $\checkmark$ & $\mathbf{x}$ \\
\hline Incorporar lenguaje audiovisual & $\checkmark$ & $\checkmark$ & $\checkmark$ \\
\hline Favorecer el feedback & $\mathbf{x}$ & $\sqrt{ }$ & $\sqrt{ }$ \\
\hline Medir resultados & $\mathbf{x}$ & $\mathbf{x}$ & $\mathbf{x}$ \\
\hline
\end{tabular}

Fuente: elaboración propia. 
En la Tabla 1, cuadro tipo check list, se realizó un contraste entre la perspectiva seleccionada de comunicación responsable y las frases testimoniales que surgieron de las entrevistas realizadas al personal de jefaturas, mandos medios y personal operativo. En los testimonios se identificaron las declaraciones útiles para contrastar la percepción de la persona entrevistada sobre determinada situación laboral, en relación con el sector comunicacional, y se encontraron declaraciones que clasificamos mediante escalas semánticas, lo que permitió hallar las discrepancias entre los preceptos establecidos por la perspectiva de comunicación responsable y la práctica de comunicación en las diferentes áreas y entre el personal de la organización escolar estudiada (ver Tabla 1).

\section{Conclusiones y proyecciones}

El objetivo planteado en esta investigación fue reconocer las discrepancias entre los preceptos establecidos por la perspectiva de comunicación responsable y la práctica comunicativa de una organización educativa.

Como se sabe, la comunicación es una acción que implica la emisión de un mensaje, y al mismo tiempo cierta reciprocidad (Oyarvide-Ramírez, Reyes-Sarria y Montaño-Colorado, 2017; Ramírez y Hugueth, 2017). En las organizaciones, la comunicación resulta una herramienta indispensable debido a que permite a sus miembros informar sobre las actividades que llevan a cabo distintos grupos de interés, recibir información y mantener una retroalimentación (interactividad) constante, propiciando flujos de información responsable que benefician el trabajo efectivo en su interior (Vásquez-Rizo y GabalánCoello, 2015; Rodríguez-Cruz y Pinto, 2018).

Por tanto, es recomendable que las organizaciones lleven a cabo una comunicación responsable en la práctica, y no solo se refleje de manera teórica, para así obtener un diálogo fluido con sus grupos de interés, definir el mensaje que se desea emitir, tener claridad sobre hacia quién va dirigido, qué se quiere lograr y por qué canales se va a transmitir, entre otros aspectos más, para que la organización logre desempeñarse con responsabilidad en ambientes comunicativos que tienen la característica de ser en alto grado competitivos. Al mismo tiempo, ello contribuirá a que puedan atenderse las expectativas y necesidades de sus públicos y a crear vínculos más sólidos a través del soporte y la práctica de una comunicación responsable constante y efectiva. En este sentido, se ubica un área de oportunidad para la organización objeto de estudio, pues, como señalan Meseguer, García y Hernández (2018), es preciso alinear los objetivos de comunicación para permitir un flujo de información pertinente y responsable entre los distintos niveles administrativos.

Sobre los resultados obtenidos en la investigación, cabe destacar que existe un gran desconocimiento por parte de la organización caso de estudio acerca de la importancia que tiene, a nivel organizacional, la práctica consciente y disciplinada de la comunicación responsable mediante vías oficiales. Contrariamente a un ideal teórico, en esta organización se considera que es suficiente con hacer comunicados en redes sociales, y no se considera valioso que sus grupos de interés no se acerquen a estos medios de comunicación digitales; para la organización, es suficiente con este tipo de comunicados y se ignora la falta de responsabilidad de sus usuarios sobre el empleo de estos espacios. El correo electrónico institucional se utiliza, sí, aunque solo para comunicar asuntos de interés interno y no para dar a conocer objetivos organizacionales, logros o la situación actual de la universidad. Habría que replantearse este uso porque, como menciona Arribas (2018), los recursos electrónicos facilitan una gran cantidad de procesos en términos de ubicuidad y son también medios importantes para la difusión de la cultura y 
las necesidades de la empresa o la organización estudiada porque la información puede llegar al receptor de manera casi inmediata.

Aunado a lo anterior, y de acuerdo con el análisis que se llevó a cabo, consideramos que es importante para la organización realizar y fomentar un manejo consciente de sus sistemas de comunicación, aplicando acciones como la segmentación, la participación y la medición de resultados, ya que, como asevera Hernández-Flores (2018), el diálogo continuo con los públicos de la institución o empresa, sea pública o privada, económica o sin fines de lucro, fortalece los lazos entre los individuos que integran la entidad y pone a prueba el compromiso y la confianza entre quienes conforman la organización en el mediano plazo. Entonces, sin importar el tipo de organización de que se trate, este es un curso de acción que debiera ser atendido para obtener resultados positivos en distintas etapas, que requieren una estrategia de comunicación adecuada para contrastar los elementos en los que la organización aún funciona de forma deficiente.

En concordancia con los resultados deseables de acuerdo con la metodología diseñada por ISEOR para encontrar costos ocultos en una organización, se detectó que algunos de los ámbitos que son susceptibles de ser mejorados por la Unidad Académica Cozumel de la Universidad de Quintana Roo, considerando los resultados, son: mejorar de la integración de las operaciones; mejorar de la comunicación y la calidad informativa; efectuar una definición más clara de las responsabilidades; reducir los costos por rechazos, desperdicios y reprocesos, y prevenir problemas bajo un esquema de comunicación responsable.

En el ámbito específico de la comunicación y la calidad informativa conviene, además, preguntarse en qué medida la falta de praxis comunicativa alcanza al resto de los grupos de interés de la organización, es decir, al personal académico y al alumnado.

Este proyecto de investigación alcanzó su objetivo en tanto que se contrastaron los resultados de las entrevistas que se le realizaron al personal gestor de la organización estudiada, con información documental sobre comunicación responsable, entre otras acciones, para hacer notar la importancia de esta práctica en la organización y establecer los ámbitos a mejorar con la intención de elevar la calidad de los servicios que proporciona. Como resultado de la investigación, se concluye que la unidad académica de la universidad analizada debe aplicar un plan de comunicación responsable que integre estrategias para propiciar mejores flujos de comunicación, tanto a nivel interno como externo, de tal forma que se facilite la información adecuada para el quehacer organizacional, para lograr que los mensajes lleguen a tiempo, para que el personal pueda atender las necesidades de sus usuarios y usuarias, y para que todas las personas puedan desempeñar con mayor eficiencia las funciones para las que fueron contratadas. No hay que perder de vista que toda organización escolar, por su carácter, se encuentra inmersa en un sistema complejo y que demanda el cumplimiento de diversos objetivos y funciones que son de gran importancia para la sociedad. Por eso, habría que hacer más simple y confiable el esquema de comunicación que conecta a este tipo de organizaciones.

Finalmente, es importante señalar que en la universidad objeto de estudio hay algunos otros elementos de comunicación que será importante tratar en futuras investigaciones, como la autoridad en el sistema comunicativo, la comunicación cara a cara, los documentos que se utilizan en la comunicación interna y cómo se ejerce el poder mediante la práctica comunicacional. 


\section{Referencias}

Arribas, A. (2018). Intranet para la gestión del conocimiento y la comunicación interna. Razón y Palabra, 22(1100), 406-413. https://www.revistarazonypalabra.com/index.php/ryp/article/view/1163

Capriotti, P. (1999). Planificación estratégica de la imagen corporativa. Ariel.

Dörnyei, Z. (2007). Research methods in applied linguistics: Quantitative, qualitative, and mixed methodologies. Oxford University Press.

Fernández, M., y Savall, H. (2004). El modelo de gestión socio-económica en organizaciones mexicanas. Universidad Autónoma Metropolitana-Iztapalapa.

Fuentes, O. (2015). La organización escolar. Fundamentos e importancia para la dirección en la educación. VARONA, Revista Cientifico-Metodológica, (61), 1-12. https://www.redalyc.org/pdf/3606/360643422005.pdf

Fundación SERES (2014, 6 de noviembre). 10 consejos para comunicar con éxito la RSE. Compromiso RSE. https:/www.compromisorse.com/rse/2014/11/06/10-consejos-para-comunicar-con-exito-la-rse-/

Garbanzo-Vargas, G. (2016). Desarrollo organizacional y los procesos de cambio en las instituciones educativas, un reto de la gestión de la educación. Revista Educación. 40(1), 67-87. http://dx.doi.org/10.15517/revedu.v40il.22534

Hernández Flores, H. G. (2018). De la comunicación institucional a la responsable, un beneficio auténtico para las organizaciones. ObservaRSE. http://www.observarse.com/2018/01/04/comunicacion-institucionalresponsable-beneficio-organizaciones/

Instituto de Socioeconomía de las Empresas y Organizaciones (ISEOR) (s.f.). El modelo de gestión socioeconómica en organizaciones mexicanas. http://164.132.163.82/SiteIseor/ouvrages_fiche.asp?code=LIV2004MFE001

Jaksa, J. (1996). Responsible Communication: Ethical Issues in Business, Industry, and the Professions. Hampton Press.

Meseguer, J., García, M., y Hernández, R. (2018). Manual de comunicación creativa y responsable. Universidad Católica San Antonio, Lógica Ecommerce. https:/logicaecommerce.com/formacion/wp-content/ uploads/2018/12/guia-de-la-comunicacion-responsable-final.pdf

Orozco-Toro, J., y Ferré-Pavia, C. (2019). Los intangibles de la marca y su efecto en la reputación corporativa. La evaluación de toda cadena de valor de Televisión de Catalunya. Revista de Comunicación, 18(1), 111-134. https://doi.org/10.26441/RC18.1-2019-A6

Oyarvide-Ramírez, H., Reyes-Sarria, E., y Montaño-Colorado, M. (2017). La comunicación interna como herramienta indispensable de la administración de empresas. Revista Científica Dominio de las Ciencias, 3(4), 296-309. http://dx.doi.org/10.23857/dc.v3i4.687

Paladines, F., Valarezo, K., y Yaguache, J. (2013). La comunicación integral, un factor determinante en la gestión de la empresa ecuatoriana. Signo y Pensamiento, 32(63), 110-128. https://doi.org/10.11144/Javeriana.syp32-63.cifd

Pertúz, F. (2018). Liderazgo transformacional en empresas sociales desde la perspectiva ética de la responsabilidad social empresarial. Telos, 20(2), 377-388. https://dialnet.unirioja.es/servlet/articulo?codigo=6436416

Ramírez, R., y Hugueth, A. (2017). Modelo de comunicación productiva para las organizaciones de salud pública en Venezuela. Opción, 33(83), 305-335. https://www.redalyc.org/pdf/310/31053772011.pdf

Remund, D. L. (2015). The ART of Responsible Communication: Leading with Values Every Day. Business Expert.

Rincón, Y., y Ramírez, R. (2018). Comunicación responsable en las organizaciones. En Quintero, M., y Sánchez, M. (eds.). Responsabilidad social corporativa. Una mirada integral en América Latina (pp. 93-100). Editorial Univalle. https://www.researchgate.net/publication/328160423_Comunicacion_responsable_en_las_organizaciones

Rincón, Y., Sukier, H., Contreras, J., y Ramírez, R. (2019). Estrategias de comunicación responsable para pequeñas y medianas empresas. Opción, 35(89-2), 1208-1234. https://repositorio.cuc.edu.co/handle/11323/5377

Rivera Salas, P., y Hernández Flores, H. (2020). Comunicación responsable en infografías digitales publicadas por empresas farmacéuticas. Correspondencias e Análisis, (12). https://doi.org/10.24265/cian.2020.nl2.02

Rodríguez-Cruz, Y., y Pinto, M. (2018). Modelo de uso de información para la toma de decisiones estratégicas en organizaciones de información. Transinformação, 30(1), 51-64. https://doi.org/10.1590/231808892018000100005

Tapia-Carreto, A., Mendoza, R., y Martínez, O. (2016). Reconociendo el desarrollo de competencias sociodiscursivas en la universidad. AgoradeHeterodixias, 2(2), 22-36. https://redib.org/Record/oai_articulo2905109reconociendo-el-desarrollo-de-competencias-socio-discursivas-en-la-universidad

Vásquez-Rizo, F.-E., y Gabalán-Coello, J. (2015). Información y ventaja competitiva. Coexistencia exitosa en las organizaciones de vanguardia. Profesional de la Información, 24(2), 149-156. https://doi.org/10.3145/epi.2015.mar.08

Villalba, J. (2015, 9 de marzo). Comunicación responsable: una oportunidad para ganar. Telefónica Empresas. https://empresas.blogthinkbig.com/comunicacion-responsable-una-oportunidad-para-ganar/ 